\title{
NUEVO MERCADO NACIONAL DE GANADO
}

\author{
(NEW NATIONAL LIVESTOCK MARKET)
}

\author{
Alejandro Sánchez de Dios, Ingeniero Agrónomo \\ Eido Galicia, S. L. A Coruña
}

ESPAÑA

Fecha de recepción. 7-VI-95

\section{RESUMEN}

El nuevo Mercado Nacional de Ganado de Santiago de Compostela nace como consecuencia de la necesidad de trasladar las actuales edificaciones, obsoletas y mal ubicadas, a un nuevo recinto, que estará dotado de mejores accesos, mayor espacio para estacionamiento y circulación de vehiculos y más modernas y completas instalaciones. Se pretende además que sirva como recinto ferial de carácter general.

A lo largo del artículo se aborda una descripción somera de los elementos más significativos de la nueva instalación, desde la edificación principal, de $17.000 \mathrm{~m}^{2}$, con estructura de madera laminada de $53 \mathrm{~m}$ de luz, hasta el equipamiento informático, uno de los más completos de Europa en este tipo de instalaciones

\section{SUMMARY}

The new National Livestock Market of Santiago de Compostela is the result of the need to move the present obsolete and badly located buildings to a new place which will have better access roads, better parking and circulation space for vehicles and more modern and complete installations. The complex is also meant to be a generalpurpose fairground.

The article briefly describes the most important elements of the new installation, including the main $17,000 \mathrm{~m}^{2}$ building, with a laminated wood structure of a $53 \mathrm{~m}$ span, and the computer equipment which is one of the most complete ones in Europe in this type of installations.

\section{Un nuevo Mercado para la ciudad de Santiago}

(Figs. 1 y 2)

Un rasgo característico del pasado de la ciudad de Santiago, enmascarado quizá por su relevancia como eje cultural y religioso, ha sido su importancia como centro comercial agrícola y ganadero, por el que han tenido entrada no pocas de las innovaciones tecnológicas aplicadas a lo largo del tiempo en las comarcas centrales de la Galicia rural. Históricamente, el mercado de ganado que se celebraba en la carballeira de Santa Susana, dio paso, en 1971, al actual Mercado Nacional de Ganado, situado en el barrio de Salgueiriños, que, con una concurrencia de 250.000 cabezas de ganado y un volumen de transacciones que alcanza 


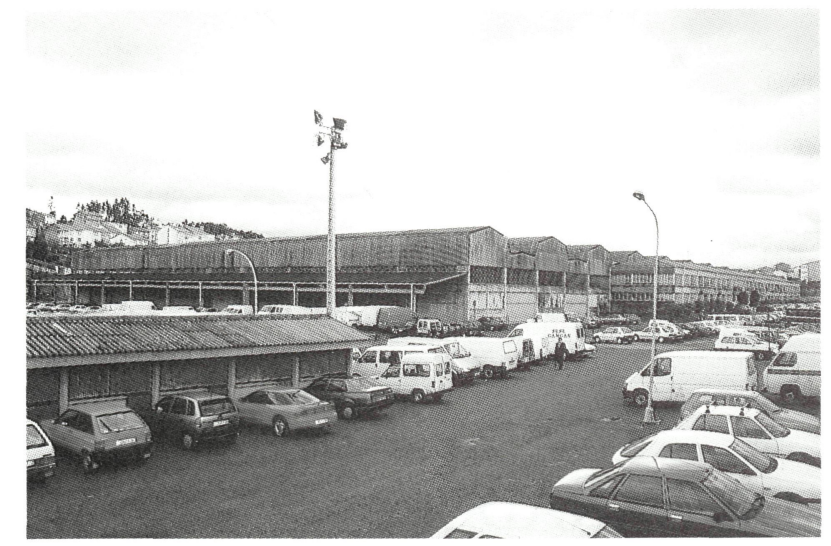

Fig. 1.- El actual Mercado de Ganado.

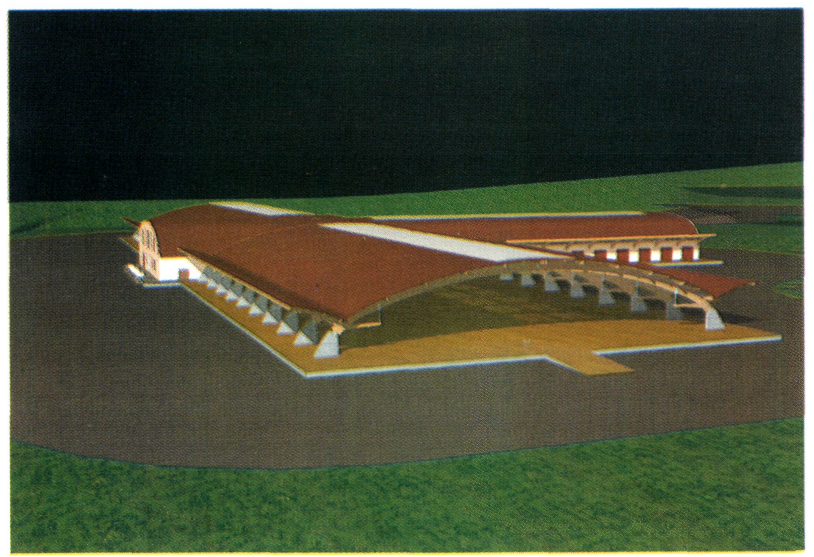

Fig. 2.- Vista general de la nueva instalación.

los 15.000 millones de pesetas anuales, constituye el centro comercial ganadero más importante de Galicia y el segundo del Norte peninsular.

Sin embargo, sus instalaciones, inauguradas en lo que en su momento era un emplazamiento extrarradial, ocupan hoy un espacio privilegiado dentro de los límites de crecimiento natural de la ciudad, para el que se prevén usos residenciales y terciarios. Si a ello se añade la obsolescencia y mal estado de las edificaciones, el negativo impacto visual que prestan a una de las entradas principales de la ciudad y los problemas de tráfico que la afluencia de vehículos de transporte de ganado ocasiona en un área tan próxima al centro urbano, se comprenderá que su traslado constituye una necesidad insoslayable y urgente.

Ahora bien, la ciudad de Santiago no se puede plantear la desaparición de laactividad ligadaal Mercado, no solamente por su indudable importancia económica, sino también -y fundamentalmente- porque constituye el pilar básico del carácter integrador del binomio campo-ciudad que Compostela ha presentado a lo largo de su historia. El proceso de metropolización en el que la ciudad se halla

(c) Consejo Superior de Investigaciones Científicas Licencia Creative Commons 3.0 España (by-nc) inmersa, y del que forman parte las grandes actuaciones urbanísticas, dotacionales y de infraestructuras que se están llevando a cabo, ha de asimilar las actividades tradicionales en el conjunto de la generación de pequeña y mediana industria ligada a las nuevas tecnologías, en el que deben tener cabida las industrias creadoras de insumos a la agricultura y las transformadoras de productos agrarios y ganaderos. El nuevo Mercado deberá por tanto retomar la tradición de la ciudad como puerta de relación con su entorno, utilizando la nueva dotación como elemento dinamizador para el sector, que sirva de marco para la realización de actividades que impliquen intercambio de conocimientos, experiencias y tecnología.

Finalmente, parece un contrasentido el destinar unas inversiones que rondarán, si se tiene en cuenta el coste de los terrenos, los 2.500 millones de pesetas, exclusivamente a la celebración de una actividad ferial ganadera que, por importante que sea, solamente tiene lugar una vez a la semana, por lo que se pretende que, sin perder su funcionalidad, las nuevas instalaciones puedan permitir la celebración de certámenes feriales no directamente relacionados con el sector, esto es, constituir el germen de un recinto ferial de carácter general.

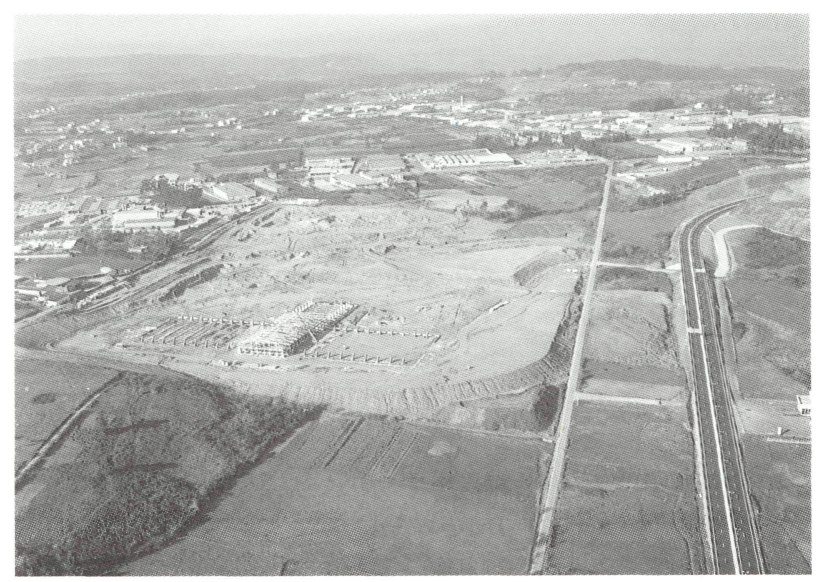

Fig. 3.- Vista aérea del estado de las obras (marzo 1995).

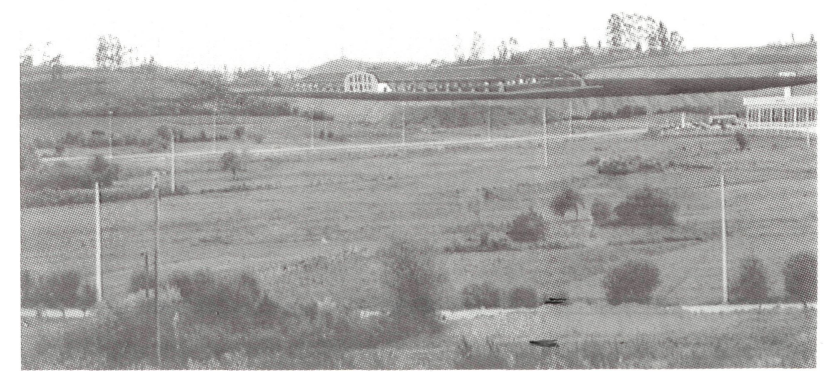

Fig. 4.- Vista principal desde la Autopista del Atlántico. 


\section{Localización (Figs. 3 y 4 )}

El nuevo Mercado está siendo construido en una parcela de $180.000 \mathrm{~m}^{2}$ de superficie total, situada al NE. del casco urbano, y contará con acceso directo a la carretera $\mathrm{N}-525$, que enlaza Santiago con A Coruña, al Periférico y a la nueva autovía del Aeropuerto. Se trata por tanto de un emplazamiento bien integrado en la red de comunicaciones, al tiempo que debidamente alejado del casco urbano. La parcela presenta una pendiente media superior al $5 \%$ y orientación Sur, con alineación de la fachada principal hacia los accesos a la ciudad por la Autopista del Atlántico, Periférico y autovía de Labacolla.

\section{Dimensión (Fig.5)}

Se proyecta la construcción de un conjunto de edificaciones con una superficie total en planta de $17.712 \mathrm{~m}^{2}$, capaces de albergar simultáneamente un total de 5.000 cabezas de ganado. La superficie total urbanizada será de $131.705 \mathrm{~m}^{2}$, de los que $111.990 \mathrm{~m}^{2}$ se destinarán a estacionamiento de vehículos (con capacidad para 750 camiones y 625 turismos), a plazas de carga y descarga (un total de 146) y a viales de circulación.

\section{Objetivos generales}

El diseño del nuevo Mercado ha sido planteado atendiendo preferentemente a una serie de objetivos de carácter general

*Facilitar la manipulación de animales (adecuadas condiciones de circulación de vehículos y sistemas de carga y descarga) y favorecer el bienestar de su alojamiento (diferenciar las condiciones de alojamiento en función de las necesidades de cada especie).

* Garantizar condiciones de competencia leal y transparencia de precios, lo que implica estimular la introducción de nuevos sistemas comerciales, como pueden ser las ventas por subasta, y dotar adecuadamente a la instalación de equipamiento administrativo e informático.

* Aplicar criterios urbanísticos, arquitectónicos y medioambientales que respeten la integración de la instalación en el entorno.

*Enfocar la elección de materiales y tecnologías constructivas hacia aquéllos que permitan un bajo mantenimiento y una adecuada resistencia a la acción agresiva del medio.

*Permitir, y aún incentivar, la utilización polivalente del recinto en condiciones dignas, pero sin limitar por ello la funcionalidad del uso para el que se concibe en primer lugar.
*Cumplir lo dispuesto por las Directivas (CEE) 64/432 y 91/68, traspuestas al Derecho español por los R.D. 434/ 1990 y 679/1993, que establecen las condiciones sanitarias aplicables al comercio intracomunitario de animales vivos. Además de esto, aun cuando no se trata de normas de obligado cumplimiento, el Mercado ha sido diseñado siguiendo el inventario de criterios organizativos elaborados por la Asociación Europea de Mercados de Ganado.

\section{Definición de las alternativas elegidas (Figs. 6 y 7)}

Los objetivos generales antes establecidos exigen reservar grandes espacios para aparcamientos, circulación de vehículos y plazas de carga y descarga. Ello obliga al destino de más de $110.000 \mathrm{~m}^{2}$ para áreas asfaltadas. Pero, al tiempo, es preciso hacer compatible esta gran dotación de superficie pavimentada $-y$ su consiguiente impacto visual sobre el entorno y los puntos de vista principales- con la topografia de la parcela, caracterizada por un desnivel de más de $40 \mathrm{~m}$ entre los puntos extremos. La solución a este compromiso se ha buscado en la formación de tres grandes plataformas, con diferencias de cota de $7 \mathrm{~m}$ y taludes entre $20^{\circ}$ y $30^{\circ}$. La plataforma principal, con una superficie total de $89.000 \mathrm{~m}^{2}$, alojará las edificaciones y las áreas de acceso rodado y de carga y descarga, así como el estacionamiento de turismos. Las otras dos plataformas, de 45.000 y 16.000 $\mathrm{m}^{2}$, serán dedicadas íntegramente al estacionamiento de vehículos de transporte de animales. La plataforma principal se encuentra a cota similar a la de la rasante de la autopista en esa zona y ligeramente superior a la de los otros dos accesos, por lo que la apreciación de la vista principal es agradable y el acercamiento se produce sin sobresaltos.

La preferencia por alojar los diferentes tipos de ganado de manera diferenciada, en función de sus necesidades de alojamiento, junto con la obligación de maximizar el númerode plazas de cargay descarga, condujo a la solución de la edificacion en "T", con un núcleo de unión ocupado por las áreas administrativas y de servicios generales, del que parten tres alas. Dos de ellas son idénticas, de $5.900 \mathrm{~m}^{2}$ de superficie total, una altura máxima de $11,50 \mathrm{~m}$ y estructura formada por arcos triarticulados de madera laminada de $53 \mathrm{~m}$ de luz. Ambas naves son completamente abiertas frontal y lateralmente, y estarán destinadas al alojamiento de animales de abasto, que no requieren gran control térmico pero sí suficiente ventilación. La tercera ala es de menor tamaño, con $3.630 \mathrm{~m}^{2}$ de superficie, y asimismo con estructura de madera laminada de $34,50 \mathrm{~m}$ de luz y $11,50 \mathrm{~m}$ de altura. Esta nave estará destinada a alojar animales de recría, de corta edad, con mayores exigencias de aislamiento térmico, y estará completamente cerrada y dotada de instalaciones de ventilación forzada.El núcleo de encuentro entre las naves, con $2.670 \mathrm{~m}^{2}$ de superficie total dividida en dos plantas, alojará, en planta baja,entre otras dependencias,las oficinas de administración, 


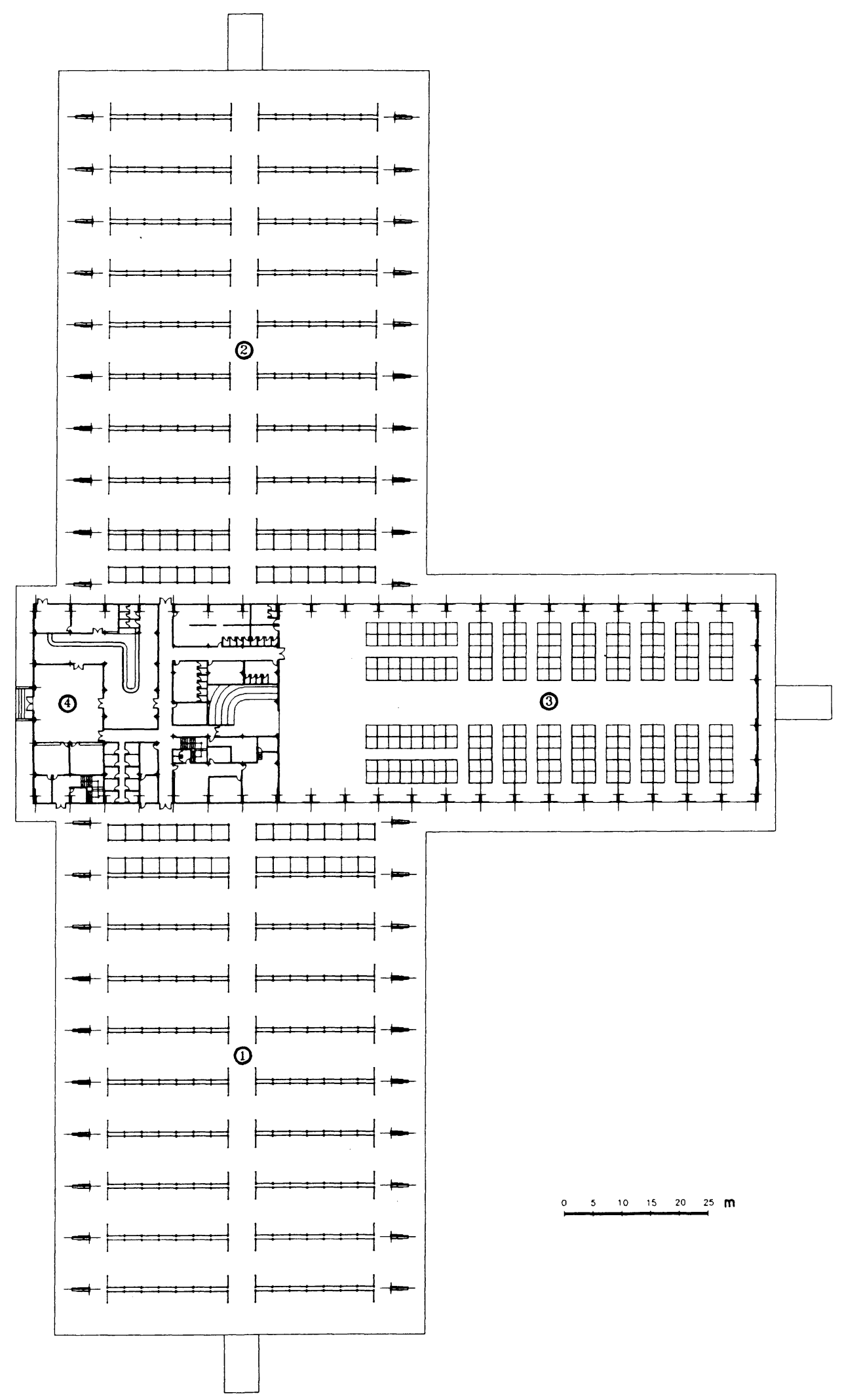

Fig. 5.- Planta general: 1) Estabulación ganado mayor de abasto. 2) Estabulación ternero de abasto. 3) Estabulación ternēro de recría. 4) Edificio administrativo y de servicios.

$-$ 

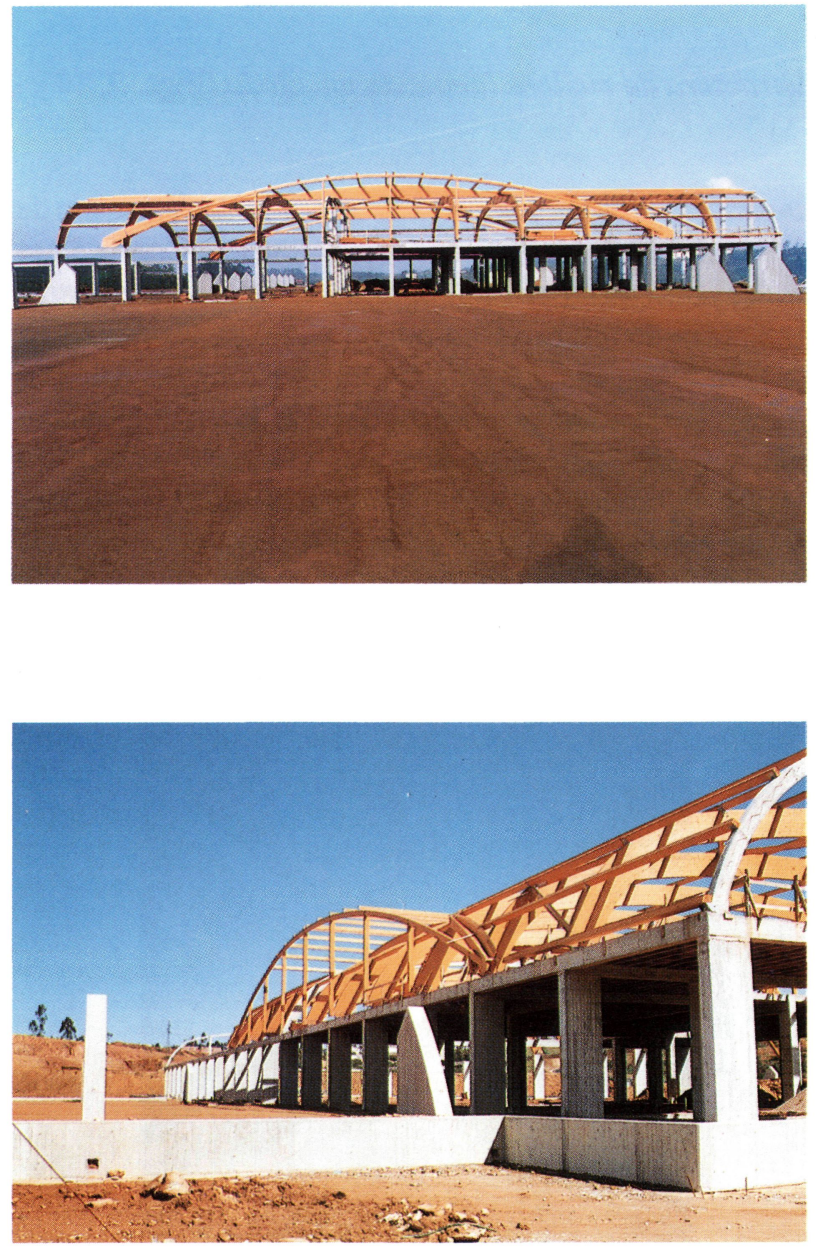

Figs.6 y 7.- Estructura del edificio de servicios.

cafetería, locales bancarios y un gran vestíbulo abierto a la planta alta que, a su vez, alojará las dependencias de la gerencia, salón de actos, salas de conferencias y un gran área de visitas, con vistas a las tres naves y al anillo de subastas.
Esta distribución no sólo no dificulta, sino que facilita los usos feriales alternativos del recinto, al permitir la distribución en distintos usos de las tres naves, y reducir la distancia máxima a la zona común de servicios.

\section{Descripción de los elementos singulares}

A continuación se procede a detallar someramente aquellos aspectos constructivos ode instalaciones que presentan algún tipo de singularidad, ya sea por la especificidad de su función o por la solución elegida.

\section{Formación de las plataformas}

El control de la ejecución del movimiento de tierras ha sido especialmente exhaustivo, teniendo en cuenta los volúmenes que se han empleado en la formación de las tres plataformas $\left(590.000 \mathrm{~m}^{3}\right.$ de excavación y $250.000 \mathrm{~m}^{3} \mathrm{de}$ rellenos) y el carácter heterogéneo que presenta el terreno. Así, en el Plan de Control de Calidad, se ha dispuesto la permanencia de un técnico a pie de obra durante los trabajos de terraplenado y conformación de explanadas. Veinticinco ensayos de identificación y clasificación de materiales en Laboratorio (determinaciones granulométricas, límites de Atterberg, Proctor Normal, índice C.B.R. y contenido de materia orgánica), y 800 determinaciones "in situ" del grado de compactación por el método de isótopos radiactivos realizados hasta la fecha (con un $80 \%$ del movimiento de tierras ejecutado) dan idea del grado de control llevado a cabo sobre estos trabajos.

\section{Cimentación (Fig. 8)}

A la hora de ejecutar la cimentación de la edificación principal, ha sido preciso resolver simultáneamente las siguientes cuestiones :

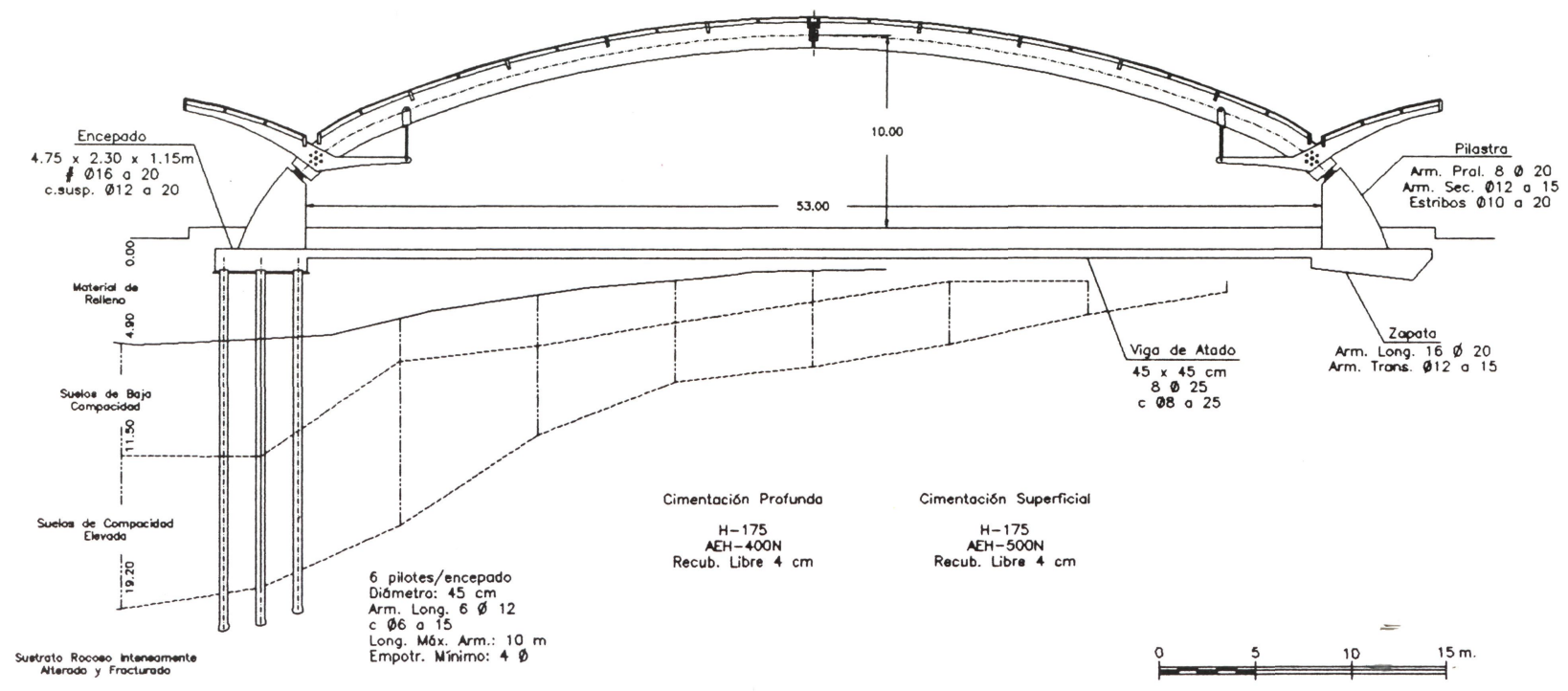

Fig. 8.- Sección esquemática por un arco de la nave principal. 
*Una de las hiladas de pilastras se apoya sobre una zona que está desmontada, en la que las solicitaciones de proyecto $\left(2,5 \mathrm{~kg} / \mathrm{cm}^{2}\right.$ de tensión admisible $\mathrm{y} 1 \mathrm{~cm}$. de asiento máximo) se alcanzan sobradamente a la cota de formación de la plataforma, y la otra hilada sobre una zona de relleno de hasta $7 \mathrm{~m}$ de altura, a la que hay que añadir, de acuerdo con los resultados de los ensayos geotécnicos, hasta $12 \mathrm{~m}$ de espesor de suelos de baja y media compacidad, procedentes de un sustrato rocoso fuertemente alteradoy de baja calidad, con aparición puntual de capas intermedias de compacidad extremadamente baja.

*Las acciones transmitidas por la estructura representan unos esfuerzos verticales de $37 \mathrm{t}$ y horizontales de 53,5 t. La importancia de estos últimos es obvia, máxime si se tienen en cuenta las deficientes condiciones geotécnicas que presenta la parcela.

La cimentación de la edificación principal ha sido resuelta mediante formación de un sistema mixto de cimentación superficial-profunda, caracterizado por

*La búsqueda de la alineación estructural de modo que una de las hiladas de pilastras se apoyase sobre zapatas superficiales de $6,30 \times 2,00 \times 1,60 \mathrm{~m}$, y la otra sobre encepados de $4,75 \times 2,30 \times 1,15 \mathrm{~m}$, sobre grupos de seis pilotes cada uno, ejecutados in situ por el método de perforación con barrena continua y hormigonado por tubo central, con diámetros de $45 \mathrm{~cm}$ y profundidades que oscilan entre 14 y $22 \mathrm{~m}$. La longitud total de pilotes ejecutados ha sido de $3.670 \mathrm{~m}$. Esta solución permite absorber-sobradamente los esfuerzos verticales transmitidos por la estructura.

*Como solución para absorber los esfuerzos horizontales, se tanteó, en primer lugar, la posibilidad de absorción de los esfuerzos, en el caso de las zapatas, por su resistencia a deslizamiento, complementada con la formación de tacones, y en el caso de los pilotes, dimensionando los armados para absorber estos esfuerzos, al asimilar los pilotes a ménsulas equivalentes de análogas características mecánicas, sometidas sólo a las fuerzas exteriores y en las que la acción del suelo se transformase en un empotramiento estructural ficticio, de acuerdo con el método desarrollado por C. Oteo. Sin embargo, la falta de garantías que la heterogeneidad del terreno de asiento provoca, tanto en la determinación del ángulo de rozamiento interno como de su coefíciente de reacción lateral, hizo abandonar esta solución. Tras comprobar otras posibilidades -como la realización de un atirantado postesado del sistema zapataencepado o la contribución de la resistencia a rozamiento de la solera, mediante el anclaje de las zapatas a la misma-, se ha optado por una solución más conservadora, pero que garantiza más fehacientemente la durabilidad, sobre todo teniendo en cuenta la naturaleza de los posibles elementos filtrados (deyecciones animales y productos de limpieza), solución consistente en el atirantado mediante riostras dimensionadas para trabajar a unas tensiones inferiores a
Estructura de madera laminada encolada (Figs. 9, 10 y 11)

La estructura de las dos naves de alojamiento de ganado de abasto está formada por unos arcos triarticulados de $53 \mathrm{~m}$ de luz y $9 \mathrm{~m}$ de separación, apoyados sobre unas pilastras de 3,50 $\mathrm{m}$ de altura de hormigón visto, en las que se ha querido mantener continuidad en la curvatura del arco hasta el suelo. Las marquesinas de estas naves se sustentan por medio de elementos articulados en los extremos de cada arco, en los que las resultantes verticales de las acciones se transmiten al conjunto de la estructura por medio de barras de acero galvanizado.
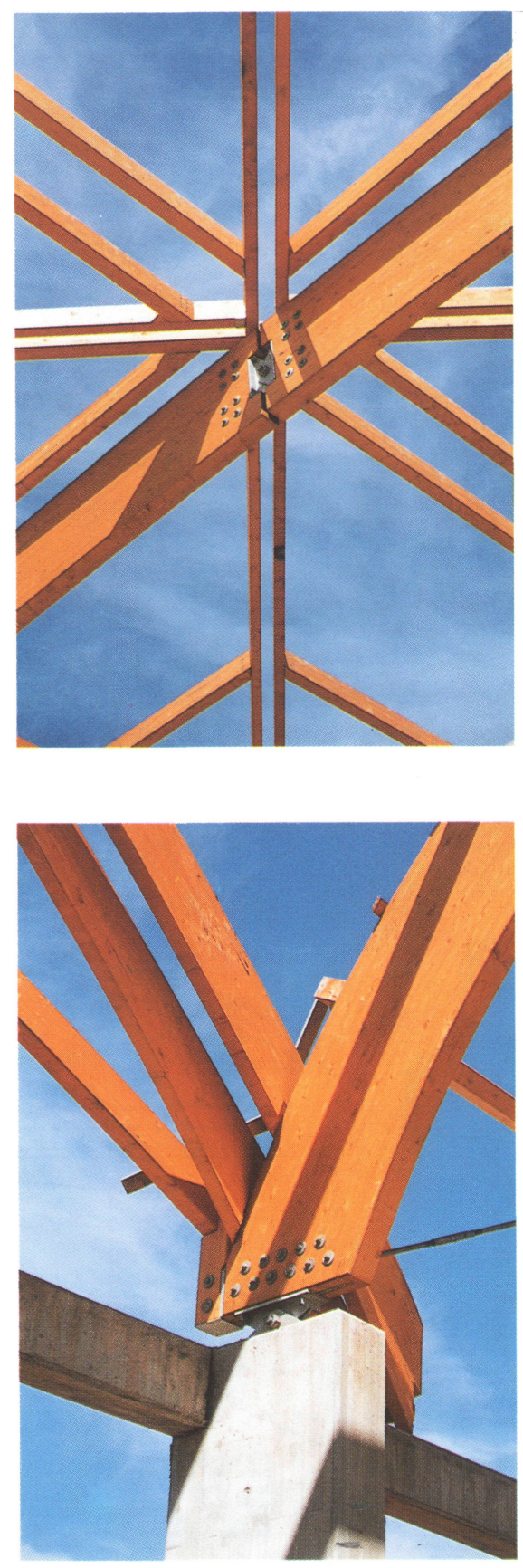

Figs. 9 y 10.-Detalles de la estructura de madera laminada.

http://informesdelaconstruccion.revistas.csic.es 


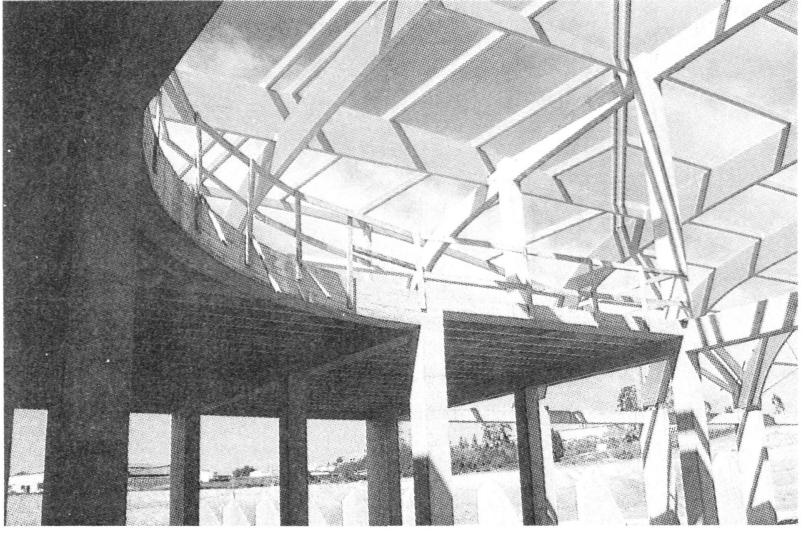

Fig. 11.- Estructura del anillo de subastas.

La nave de ganado de recría está formada por arcos igualmente triarticulados, pero en este caso de $34,5 \mathrm{~m}$ de luz. Los dos tercios centrales del arco mantienen la mis ma curvatura que los de las otras naves, con el fin de permitir la formación de un encuentro regular entre ellas. En los extremos se fuerza sin embargo la curvatura para conseguir reducir la luz total. En este caso, al tratarse de una nave completamente cerrada, las pilastras de apoyo, igualmente de hormigón visto, tienen forma paralelepipédica, con el fin de facilitar la formación del cerramiento; y, además, porque la directriz del arco en el apoyo es ya vertical. Las marquesinas se empotran en las pilastras y son atirantadas a los arcos mediante cables de acero inoxidable.

Para la realización del cálculo, se ha seguido lo establecido en la Norma DIN 1.052 "Estructuras de madera. Cálculo y construcción". En cuanto ala determinación del fabricante, se ha exigido que éste estuviese cualificado con el certificado del grupo A del Instituto Otto Graf (Alemania) o con el grado de tecnicidad 3 del OPQCB (Francia). Y finalmente, en lo referente al control de calidad de fabricación, además de los autocontroles del fabricante determinados por las cualificaciones del sello de calidad, se han seguido las especificaciones incluidas en las normas UNE EN 391 (ensayos de deslaminación), UNE EN 392 (ensayos de cortante) y UNE EN 408 (resistencia característica).

\section{Soleras y pavimento (Fig. 12)}

Las soleras de las naves principales, con una superficie total de $17.000 \mathrm{~m}^{2}$, totalmente diáfanas, van a servir de soporte al entramado de estructuras de amarre. Para conseguir una buena resistencia a flexotracción y una mínima aparición de fisuras de retracción, se ha optado por la formación de una solera de $18 \mathrm{~cm}$ de hormigón $\mathrm{H}-175$ armada con grapillas de acero de dimensión 30/50 en dosificación de $20 \mathrm{~kg} / \mathrm{m}^{3}$.

El pavimentode las naves de estabulación ha de cumplir un doble objetivo:

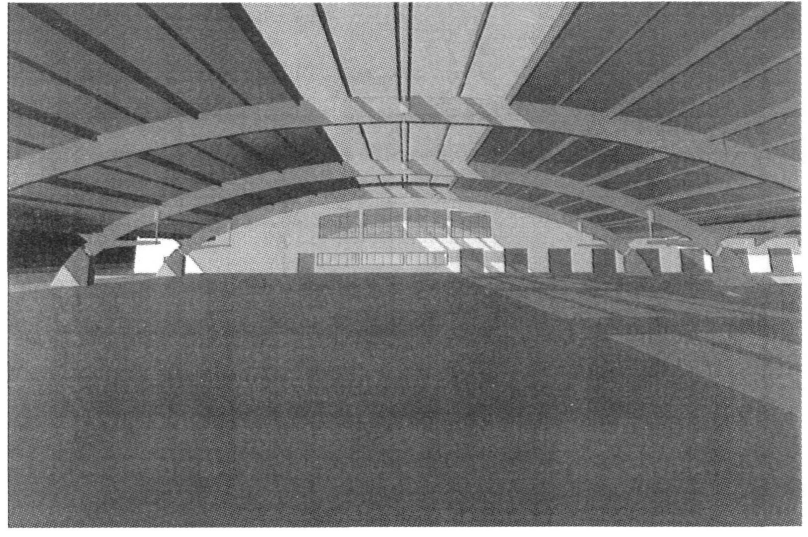

Fig. 12.- Vista interior de la nave principal.

* Debe tratarse de un material con buena resistencia química, capaz de soportar la acción de las deyecciones del ganado y la de los productos de limpieza.

*Tiene que combinar dos cualidades cuasi antagónicas, como son un cierto grado de antideslizamiento, para evitar las resbalones de ganado y personal en condiciones de extrema suciedad, y la capacidad de rápida limpieza y desinfección, para conseguir una conversión eficaz del uso ganadero al ferial.

Se ha elegido un tipo de pavimento muy empleado en la industria alimentaria, pero novedoso para usos ganaderos, como es un revestimiento a base de resinas epoxídicas exentas de disolventes, combinada con agregados minerales de alta dureza (sílice y corindón), con el fin de presentar alta resistencia química la acción de los ácidos minerales, bases, disolventes y ácidos orgánicos diluidos y acabado antideslizante. Para conseguir un producto capaz de equilibrar el acabado antideslizante y la facilidad de limpieza, se ha recurrido a la aplicación de 10 muestras con diferentes dosificaciones en el Mercado actual, en lugares de fuerte tránsito, y con ello poder elegir la dosificación de resina-árido que se considere óptima.

Amarres móviles (Fig. 13)

El sistema de amarres previsto para el ganado ha de cumplir una serie de objetivos :

*Debe ser fácilmente desmontable, con el fin de que en breve espacio de tiempo se puedan destinar las naves a los usos alternativos.

*Ha de ser funcional, esto es, debe permitir cumplir su misión con las mejores condiciones de confort e higiénicas.

*Tiene que estar constituido por pocos elementos distintos, de fácil identificación y de montaje sencillo. 


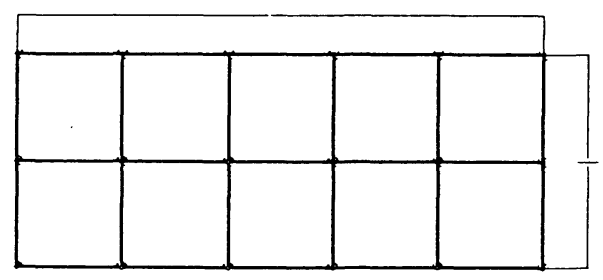

DISTRIBUCIÓN STANDARD DE MODULOS EN NAVE DE RECRIA $\quad$ E. $1: 26$

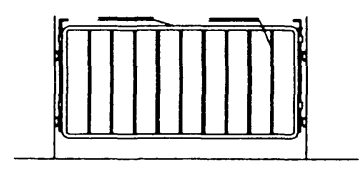

DETALLE DE MÓdULO A PARA GANADO OVINO
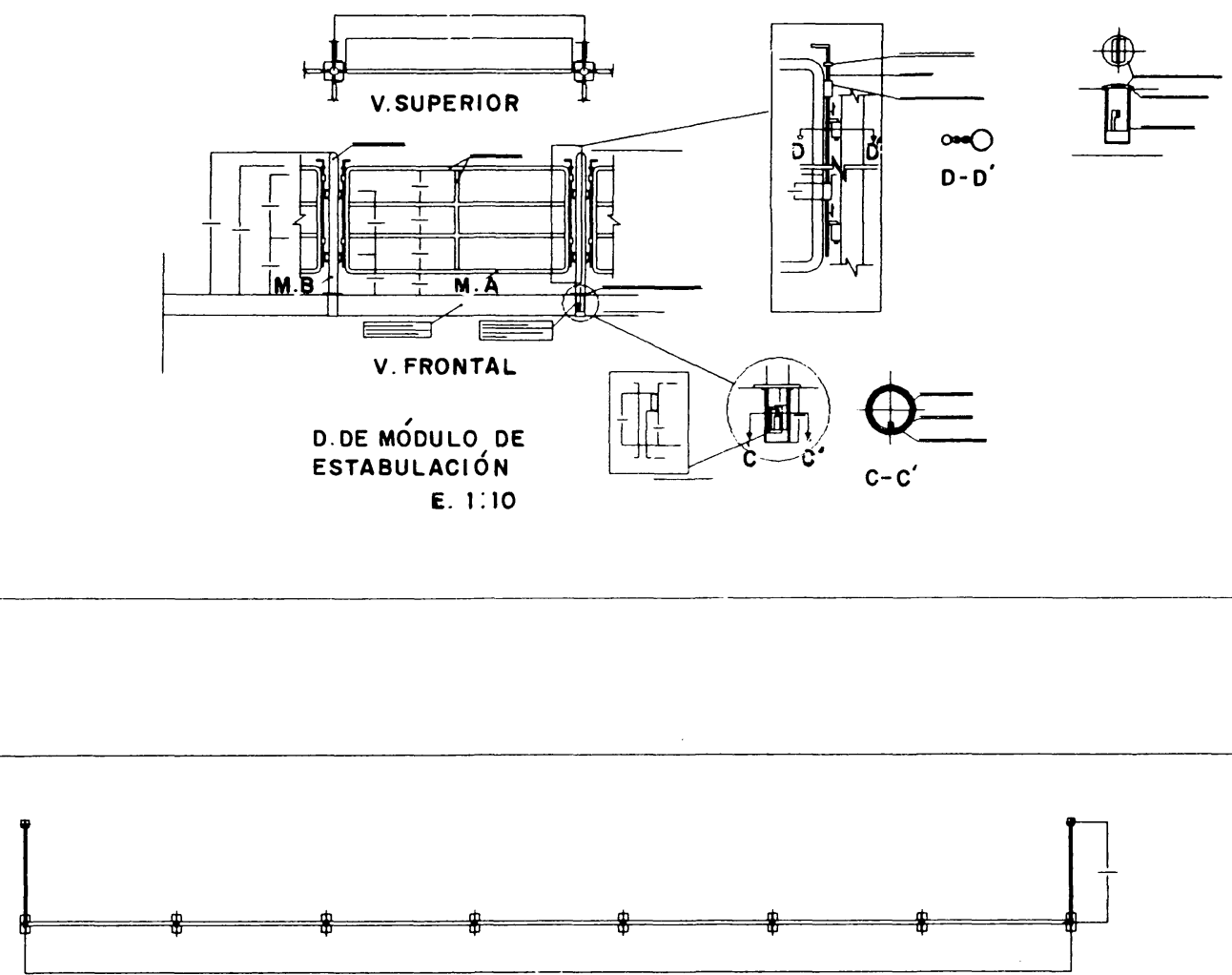

DISTRIBUCIÓN STANDARD DE MÓdULOS EN NAVES DE ABASTO E. 1:25
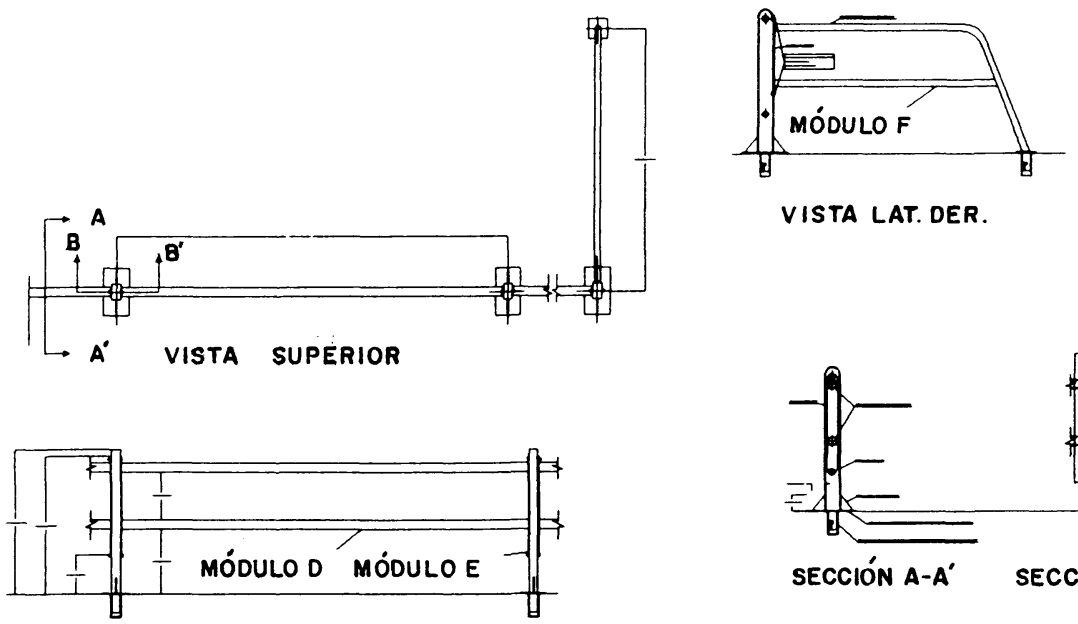

VISTA LAT. DER.

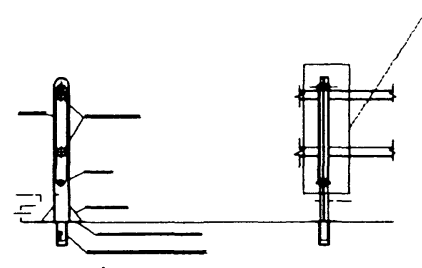

SECCIÓN A-A' SECCIÓN B-B'

DETALLE de MÓdULO de AMARRE E.1:10 
*Debe ser mecánicamente estable, pese a su facilidad de montaje.

*Ha de ser resistente a las acciones ocasionadas por los animales y a la acción del medio.

Se ha previsto la instalación de una serie de módulos de amarre en acero galvanizado, de diseño propio, algunos de cuyos elementos se recogen en los detalles adjuntos. Para el anclaje de estos elementos, se ha previsto el siguiente sistema: una vez ejecutada la solera y fratasada ésta, se procede al replanteo con métodos taquimétricos de los diferentes anclajes, al taladrado de la solera con una broca de $110 \mathrm{~mm}$, y a la colocación de los anclajes con resina epoxi. Tanto la longitud de los anclajes como la adherencia mínima han sido calculadas para resistir un esfuerzo mínimo de $1.500 \mathrm{~kg}$, provocado por la acción de un animal sobre la cabeza del soporte.

\section{Instalación de lavado y desinfección de vehiculos}

El diseño de una instalación capaz de atender a las operaciones de limpieza de un total de 700 vehículos de ganado, de un modo rápido, sencillo y funcional, constituye un factor muy importante en la calidad higiénicosanitaria del servicio. La solución prevista consiste en aprovechar la rampa de conexión entre las plataformas 1 y 2 para construir una estación formada por seis carriles con un $7,5 \%$ de pendiente, en la que se realizarán consecutivamen- te las siguientes operaciones: vaciado de camas del ganado a una banda rodante de PVC que las transportará hacia un contenedor, limpieza de las cajas de los vehículos con agua a presión de $8 \mathrm{~kg} / \mathrm{cm}^{2}, y$ desinfección, por medio de grupos de bombeo con dosificador de desinfectante. Deeste modo, la estación tiene una capacidad de 18 vehículos simultáneos, y al mismo tiempo se obliga los vehículos al paso por la estación camino del aparcamiento

\section{Instalación de limpieza de las edificaciones}

Uno de los elementos clave del funcionamiento del Mercado estriba en la capacidad para realizar de un modo rápido y exhaustivo las operaciones de limpieza y desinfección de las naves, no sólo por las lógicas exigencias higiénicas. sino también para poder proceder con garantias al cambio de uso de las instalaciones, como ya se ha visto a la hora de determinar los criterios de elección de pavimento. El sistema adoptado ha consistido en la instalación de un grupo de presión centralizado, de uso exclusivo para la limpieza de las naves, y dimensionado para aportar una presión mínima de $8 \mathrm{~kg} / \mathrm{cm}^{2}$. Este grupo está conectado a un dosificador de desinfectante, de manera que, por medio de un sistema de lanzas dispuesto a lo largo de las naves, puede procederse a la limpieza a presión, a la desinfección, o a ambas, de un modo rápido y eficaz.

\section{Equipamiento informático}

Además de atender a las necesidades básicas de gestión administrativa de la instalación, tanto en sus aspectos ganaderos como feriales, la instalación informática se ha diseñado de modo que sea capaz de responder a dos aspectos fundamentales : en primer lugar, el control de concurrencia de vehículos y tipos de animales a partir de los accesos, de modo que sea posible facilitar información exacta y en tiempo real al usuario sobre el estado del mercado en un momento dado, información que será suministrada por medio de paneles informativos situados estratégicamente; y en segundo lugar, la implantación de un sistema altamente mecanizado para la realización de subastas de animales, ya sea individualmente o en lotes, y conectado con la entidad bancaria, de modo que los pagos puedan efectuarse de modo inmediato a cada operación. Para llevar a cabo estos procesos, se ha previsto la instalación de una red tipo Internet, con un servidor y doce puestos de trabajo, conectados mediante fibra óptica (Fig. 14).

\section{Ficha Técnica}

Obra: Nuevo Mercado Nacional de Ganado de Santiago de Compostela

Emplazamiento: Amio - Santiago de Compostela

Propiedad: Excmo. Ayuntamento de Santiago

Presupuesto Contrata: 1.303 .333 .643 ptas.

Financiación: Ayuntamiento de Santiago, Xunta de Galicia, FEOGA (CEE)

Proyecto: Alejandro Sánchez de Dios. Eido Galicia, S.L. A Conũ̃a

Dirección de Obra: Alejandro Sánchez de Dios, Ingeniero Agrónomo, Melchor Campoy Armero, Arquitecto Técnico y Manuel Blanco Suárez, Aparejador.

Empresa Constructora: FCC Construcciones, S.A

Estructura de Madera Laminada: Holtza, S.A. EMLE

Control de Calidad: Norcontrol, S.A.

Duración de las Obras: Agosto 1994 - septiembre 1995. 

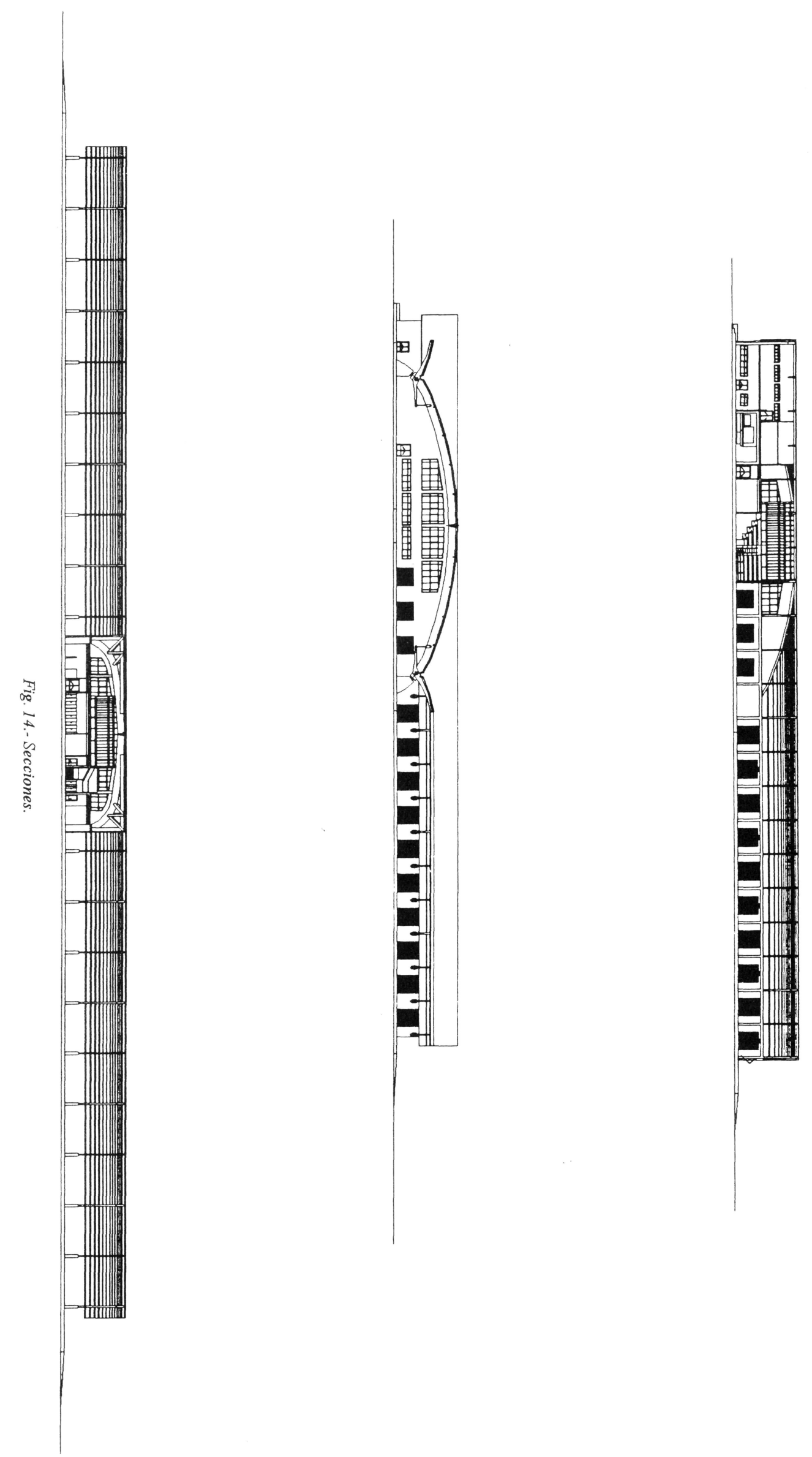